\title{
LOCALLY UNIVALENCE OF DIRICHLET SERIES SATISFYING A LINEAR DIFFERENTIAL EQUATION OF SECOND ORDER WITH EXPONENTIAL COEFFICIENTS
}

\begin{abstract}
M. M. Sheremeta. Locally univalence of Dirichlet series satisfying a linear differential equation of second order with exponential coefficients, Mat. Stud. 51 (2019), 152-158.

Let $h>0, \gamma_{0} \neq 0, \gamma_{1} \neq 0, \gamma_{2} \leq-1$ and $\left|\gamma_{1}\right| / 4+\left|\gamma_{0}\right| / 6 \leq h / 5$. It is proved that the differential equation $w^{\prime \prime}+\left(\gamma_{0} e^{2 h s}+\gamma_{1} e^{h s}+\gamma_{2}\right) w=0$ has an entire solution $F(s)=\exp \left\{s \lambda_{1}\right\}+$ $\sum_{k=2}^{\infty} f_{k} \exp \left\{s \lambda_{k}\right\}$ locally univalent in the half-plane $\{s: \operatorname{Re} s<0\}$ and such that $\ln M(\sigma, F) \sim$ $\left(\sqrt{\left|\gamma_{0}\right|} / h\right) e^{h \sigma}$ as $\sigma \rightarrow+\infty$, where $M(\sigma, F)=\sup \{|F(\sigma+i t)|: t \in \mathbb{R}\}$.
\end{abstract}

1. Introduction. An analytic univalent in $\mathbb{D}=\{z:|z|<1\}$ function

$$
f(z)=\sum_{n=0}^{\infty} f_{n} z^{n}
$$

is said to be convex if $f(\mathbb{D})$ is a convex domain. It is well known [1, p. 203] that the condition $\operatorname{Re}\left\{1+z f^{\prime \prime}(z) / f^{\prime}(z)\right\}>0(z \in \mathbb{D})$ is necessary and sufficient for the convexity of $f$. Due to W. Kaplan ([2]) the function $f$ is said to be close-to-convex in $\mathbb{D}$ (see also [1, p. 583]) if there exists a convex in $\mathbb{D}$ function $\Phi$ such that $\operatorname{Re}\left(f^{\prime}(z) / \Phi^{\prime}(z)\right)>0(z \in \mathbb{D})$. A closeto-convex function $f$ has a characteristic property that the complement $G$ of the domain $f(\mathbb{D})$ can be filled with rays $L$ which go from $\partial G$ and lie in $G$. Every close-to-convex in $\mathbb{D}$ function $f$ is univalent in $\mathbb{D}$ and, therefore, $f^{\prime}(0) \neq 0$. Hence it follows that the function $f$ is close-to-convex in $\mathbb{D}$ if and only if the function

$$
g(z)=z+\sum_{n=2}^{\infty} g_{n} z^{n},
$$

is close-to-convex in $\mathbb{D}$, where $g_{n}=f_{n} / f_{1}$. We remark also, that the function (2) is said to be starlike in $\mathbb{D}$, if $f(\mathbb{D})$ is starlike domain with respect to the origin. It is clear, that every starlike function is close-to-convex.

S. M. Shah [3] indicated conditions on real parameters $\gamma_{0}, \gamma_{1}, \gamma_{2}$ of the differential equation

$$
z^{2} \frac{d^{2} w}{d z^{2}}+z \frac{d w}{d z}+\left(\gamma_{0} z^{2}+\gamma_{1} z+\gamma_{2}\right) w=0
$$

2010 Mathematics Subject Classification: 33B50.

Keywords: Dirichlet series; locally univalence; linear differencial equation of second order. doi:10.15330/ms.51.2.152-158 
under which there exists an entire transcendental solution (1) such that $f$ and all its derivatives are close-to-convex in $\mathbb{D}$. In particular, he obtained the following result.

Theorem A. If $\gamma_{1}=\gamma_{2}=0,-2<\gamma_{0}<0$ then equation (3) has an entire solution (2) such that all derivatives $g^{(2 n+1)}(n \geq 0)$ are close-to-convex in $\mathbb{D}$, and if $\gamma_{1}=0,0<\left|\gamma_{0}\right|<2, \gamma_{2}=$ -1 then the equation (3) has an entire solution (2) such that all derivatives $g^{(2 n)}(n \geq 0)$ are close-to-convex in $\mathbb{D}$. For such solutions $\ln M_{g}(r)=(1+o(1)) \sqrt{\left|\gamma_{0}\right|} r$ as $r \rightarrow+\infty$, where $M_{g}(r)=\max \{|g(z)|:|z|=r\}$.

Substituting $z=e^{s}$ into (3) we obtain the differential equation

$$
\frac{d^{2} w}{d s^{2}}+\left(\gamma_{0} e^{2 h s}+\gamma_{1} e^{h s}+\gamma_{2}\right) w=0
$$

with $h=1$. We will consider the case, where $h$ is an arbitrary positive number and using a certain result of M.S. Robertson we will investigate the locally univalence in some half-plane of Dirichlet series with positive exponents satisfying the differential equation (4).

2. Auxiliary results. Let $0<\lambda_{1}<\lambda_{k} \uparrow+\infty$ and $f_{k} \neq 0$ for all $k \geq 2$ and a Dirichlet series

$$
F(s)=\exp \left\{s \lambda_{1}\right\}+\sum_{k=2}^{\infty} f_{k} \exp \left\{s \lambda_{k}\right\}, \quad s=\sigma+i t
$$

has the abscissa of absolute convergence $\sigma_{a} \in(-\infty,+\infty]$. Then there is the greatest real number $\tau \in\left(-\infty, \sigma_{a}\right]$, for which

$$
\sum_{k=2}^{\infty} \lambda_{k}\left|f_{k}\right| \exp \left\{\tau \lambda_{k}\right\} \leq 1
$$

Those functions $F$ given by Dirichlet series (5) which satisfy (6) is said to be of the class $\tau$. Recalling certain concepts of univalence introduced by P. Montel [4], we say that a function $f$ is locally univalent in a region $G$ if $f$ is regular in $G$ and if, for every closed domain $G^{*} \subset G$ and for every point $z_{0} \in G^{*}$, there exists a positive number $\varrho$ independependent of $z_{0}$ such that $f$ is univalent in every disk $\left\{z:\left|z-z_{0}\right|<\varrho\right\}$ lying within G. M.S. Robertson [5] proved the following theorem.

Theorem B. Let function (5) belong to the class $\tau$. If $\tau>-\frac{\ln \lambda_{1}}{\lambda_{1}}$ then $F$ is locally univalent in $\{s: \operatorname{Re} s<\tau\}$, and if $\tau \leq-\frac{\ln \lambda_{1}}{\lambda_{1}}$ then $F$ is locally univalent in

$$
\left\{s: \operatorname{Re} s<\frac{\tau \lambda_{2}+\ln \lambda_{1}}{\lambda_{2}-\lambda_{1}} \leq \tau\right\} \text {. }
$$

Now we return to differential equation (4), where $h_{0}>0, \gamma_{0} \neq 0, \gamma_{1} \neq 0$ and $\gamma_{2} \neq 0$. Substituting (5) in (4), we obtain

$$
\sum_{k=1}^{\infty} f_{k}\left(\lambda_{k}^{2}+\gamma_{2}\right) \exp \left\{s \lambda_{k}\right\}=-\sum_{k=1}^{\infty} \gamma_{1} f_{k} \exp \left\{s\left(\lambda_{k}+h\right)\right\}-\sum_{k=1}^{\infty} \gamma_{0} f_{k} \exp \left\{s\left(\lambda_{k}+2 h\right)\right\} .
$$

If $\lambda_{1}^{2}+\gamma_{2} \neq 0$ then $\left(\lambda_{1}^{2}+\gamma_{2}\right) \exp \left\{s \lambda_{1}\right\}=-(1+o(1)) \gamma_{1} \exp \left\{s\left(\lambda_{1}+h\right)\right\}, \sigma \rightarrow-\infty$, that is $\lambda_{1}^{2}+\gamma_{2}=-(1+o(1)) \gamma_{1} \exp \{s h\} \rightarrow 0(\sigma \rightarrow-\infty)$, which is impossible. Thus, $\lambda_{1}^{2}+\gamma_{2}=0$, $\gamma_{2}<0$ and $\lambda_{1}=\sqrt{-\gamma_{2}}$. Therefore, the equality (7) implies

$$
\sum_{k=2}^{\infty} f_{k}\left(\lambda_{k}^{2}+\gamma_{2}\right) \exp \left\{s \lambda_{k}\right\}=-\sum_{k=1}^{\infty} \gamma_{1} f_{k} \exp \left\{s\left(\lambda_{k}+h\right)\right\}-\sum_{k=1}^{\infty} \gamma_{0} f_{k} \exp \left\{s\left(\lambda_{k}+2 h\right)\right\} .
$$


and since $\lambda_{k}^{2}+\gamma_{2} \neq 0$, we have

$$
f_{2}\left(\lambda_{2}^{2}+\gamma_{2}\right) \exp \left\{s \lambda_{2}\right\}=-(1+o(1)) \gamma_{1} \exp \left\{s\left(\lambda_{1}+h\right)\right\}, \quad \sigma \rightarrow-\infty .
$$

Hence it follows that $\lambda_{2}=\lambda_{1}+h$ and $f_{2}=\frac{-\gamma_{1}}{\lambda_{2}^{2}+\gamma_{2}}$. But then from (8) it follows that

$$
\sum_{k=3}^{\infty} f_{k}\left(\lambda_{k}^{2}+\gamma_{2}\right) \exp \left\{s \lambda_{k}\right\}=-\sum_{k=2}^{\infty} \gamma_{1} f_{k} \exp \left\{s\left(\lambda_{k}+h\right)\right\}-\sum_{k=1}^{\infty} \gamma_{0} f_{k} \exp \left\{s\left(\lambda_{k}+2 h\right)\right\} .
$$

Hence we obtain as $\sigma \rightarrow-\infty$

$$
\begin{aligned}
f_{3}\left(\lambda_{3}^{2}+\gamma_{2}\right) \exp \left\{s \lambda_{3}\right\} & =-(1+o(1)) \gamma_{1} f_{2} \exp \left\{s\left(\lambda_{1}+2 h\right)\right\}-\gamma_{0} \exp \left\{s\left(\lambda_{1}+2 h\right)\right\}= \\
= & -(1+o(1))\left(\gamma_{1} f_{2}+\gamma_{0}\right) \exp \left\{s\left(\lambda_{1}+2 h\right)\right\}
\end{aligned}
$$

i. e. $\lambda_{3}=\lambda_{1}+2 h, f_{3}=-\frac{\gamma_{1} f_{2}}{\lambda_{3}^{2}+\gamma_{2}}-\frac{\gamma_{0}}{\lambda_{3}^{2}+\gamma_{2}}$. Then from (9) it follows that

$$
\sum_{k=4}^{\infty} f_{k}\left(\lambda_{k}^{2}+\gamma_{2}\right) \exp \left\{s \lambda_{k}\right\}=-\sum_{k=3}^{\infty} \gamma_{1} f_{k} \exp \left\{s\left(\lambda_{k}+h\right)\right\}-\sum_{k=2}^{\infty} \gamma_{0} f_{k} \exp \left\{s\left(\lambda_{k}+2 h\right)\right\},
$$

whence, as above, we obtain

$$
\lambda_{4}=\lambda_{3}+h, \quad f_{4}=-\frac{\gamma_{1} f_{3}}{\lambda_{4}^{2}+\gamma_{2}}-\frac{\gamma_{0} f_{2}}{\lambda_{4}^{2}+\gamma_{2}} .
$$

Continuing this process, we come to the formulas

$$
\lambda_{k}=\lambda_{k-1}+h, \quad f_{k}=-\frac{\gamma_{1} f_{k-1}}{\lambda_{k}^{2}+\gamma_{2}}-\frac{\gamma_{0} f_{k-2}}{\lambda_{k}^{2}+\gamma_{2}} \quad(k \geq 3) .
$$

Thus, the following statement is true.

Lemma 1. Let $h>0, \gamma_{0} \neq 0, \gamma_{1} \neq 0$ and $\gamma_{2}<0$. Then the differential equation (4) has a solution

$$
F(s)=\exp \left\{s \sqrt{\left|\gamma_{2}\right|}\right\}+\sum_{k=2}^{\infty} f_{k} \exp \left\{s \lambda_{k}\right\}, \quad \lambda_{k}=\sqrt{\left|\gamma_{2}\right|}+(k-1) h
$$

where $f_{2}=\frac{-\gamma_{1}}{\lambda_{2}^{2}+\gamma_{2}}$ and for $k \geq 3$ the coefficients $f_{k}$ are determined by recurrent formula (10).

We need also the following lemma.

Lemma 2. Let $h>0, \gamma_{0} \neq 0, \gamma_{1} \neq 0$ and $\gamma_{2}<0$. If for some $\eta \in \mathbb{R}$

$$
\frac{\lambda_{3}\left|\gamma_{1}\right|}{\lambda_{2}\left(\lambda_{3}^{2}+\gamma_{2}\right)} e^{h \eta}+\frac{\lambda_{4}\left|\gamma_{0}\right|}{\lambda_{2}\left(\lambda_{4}^{2}+\gamma_{2}\right)} e^{2 h \eta}+\lambda_{2}\left|f_{2}\right| e^{\eta \lambda_{2}}+\frac{\lambda_{3}\left|\gamma_{0}\right|}{\lambda_{3}^{2}+\gamma_{2}} e^{\eta \lambda_{3}} \leq 1
$$

then for the function (5)

$$
\sum_{k=2}^{\infty} \lambda_{k}\left|g_{k}\right| \exp \left\{\eta \lambda_{k}\right\} \leq 1
$$


Proof. In view of (10)

$$
\begin{gathered}
\sum_{k=2}^{\infty} \lambda_{k}\left|f_{k}\right| \exp \left\{\eta \lambda_{k}\right\} \leq \lambda_{2}\left|f_{2}\right| \exp \left\{\eta \lambda_{2}\right\}+ \\
+\sum_{k=3}^{\infty}\left(\frac{\lambda_{k}\left|\gamma_{1}\right|\left|f_{k-1}\right|}{\lambda_{k}^{2}+\gamma_{2}} \exp \left\{\eta \lambda_{k}\right\}+\frac{\lambda_{k}\left|\gamma_{0}\right|\left|f_{k-2}\right|}{\lambda_{k}^{2}+\gamma_{2}} \exp \left\{\eta \lambda_{k}\right\}\right)= \\
=\lambda_{2}\left|f_{2}\right| \exp \left\{\eta \lambda_{2}\right\}+\sum_{k=2}^{\infty} \frac{\lambda_{k+1}\left|\gamma_{1}\right|\left|f_{k}\right|}{\lambda_{k+1}^{2}+\gamma_{2}} \exp \left\{\eta \lambda_{k+1}\right\}+\sum_{k=1}^{\infty} \frac{\lambda_{k+2}\left|\gamma_{0}\right|\left|f_{k}\right|}{\lambda_{k+2}^{2}+\gamma_{2}} \exp \left\{\eta \lambda_{k+2}\right\}= \\
=\lambda_{2}\left|f_{2}\right| \exp \left\{\eta \lambda_{2}\right\}+\frac{\lambda_{3}\left|\gamma_{0}\right|\left|f_{1}\right|}{\lambda_{3}^{2}+\gamma_{2}} \exp \left\{\eta \lambda_{3}\right\}+ \\
+\sum_{k=2}^{\infty} \frac{\lambda_{k+1}\left|\gamma_{1}\right|\left|f_{k}\right|}{\lambda_{k+1}^{2}+\gamma_{2}} \exp \left\{\eta \lambda_{k+1}\right\}+\sum_{k=2}^{\infty} \frac{\lambda_{k+2}\left|\gamma_{0}\right|\left|f_{k}\right|}{\lambda_{k+2}^{2}+\gamma_{2}} \exp \left\{\eta \lambda_{k+2}\right\},
\end{gathered}
$$

whence

$$
\begin{aligned}
\sum_{k=2}^{\infty}(1 & \left.-\frac{\lambda_{k+1}\left|\gamma_{1}\right| \exp \left\{\eta\left(\lambda_{k+1}-\lambda_{k}\right)\right\}}{\lambda_{k}\left(\lambda_{k+1}^{2}+\gamma_{2}\right)}-\frac{\lambda_{k+2}\left|\gamma_{0}\right| \exp \left\{\eta\left(\lambda_{k+2}-\lambda_{k}\right)\right\}}{\lambda_{k}\left(\lambda_{k+2}^{2}+\gamma_{2}\right)}\right) \times \\
& \times \lambda_{k}\left|f_{k}\right| \exp \left\{\eta \lambda_{k}\right\} \leq \lambda_{2}\left|f_{2}\right| \exp \left\{\eta \lambda_{2}\right\}+\frac{\lambda_{3}\left|\gamma_{0}\right|}{\lambda_{3}^{2}+\gamma_{2}} \exp \left\{\eta \lambda_{3}\right\} .
\end{aligned}
$$

Since the sequences $\left(\frac{\lambda_{k+1}\left|\gamma_{1}\right|}{\lambda_{k}\left(\lambda_{k+1}^{2}+\gamma_{2}\right)}\right)$ and $\left(\frac{\lambda_{k+2}\left|\gamma_{1}\right|}{\lambda_{k}\left(\lambda_{k+2}^{2}+\gamma_{2}\right)}\right)$ are decreasing and $\lambda_{k+1}-\lambda_{k}=h$, we have

$$
\begin{gathered}
\sum_{k=2}^{\infty}\left(1-\frac{\lambda_{3}\left|\gamma_{1}\right| \exp \{h \eta\}}{\lambda_{2}\left(\lambda_{3}^{2}+\gamma_{2}\right)}-\frac{\lambda_{4}\left|\gamma_{0}\right| \exp \{2 h \eta\}}{\lambda_{2}\left(\lambda_{4}^{2}+\gamma_{2}\right)}\right) \lambda_{k}\left|f_{k}\right| \exp \left\{\eta \lambda_{k}\right\} \leq \\
\leq \lambda_{2}\left|f_{2}\right| \exp \left\{\eta \lambda_{2}\right\}+\frac{\lambda_{3}\left|\gamma_{0}\right|}{\lambda_{3}^{2}+\gamma_{2}} \exp \left\{\eta \lambda_{3}\right\} .
\end{gathered}
$$

From (12) it follows that

$$
\frac{\lambda_{3}\left|\gamma_{1}\right| \exp \{h \eta\}}{\lambda_{2}\left(\lambda_{3}^{2}+\gamma_{2}\right)}+\frac{\lambda_{4}\left|\gamma_{0}\right| \exp \{2 h \eta\}}{\lambda_{2}\left(\lambda_{4}^{2}+\gamma_{2}\right)}<1
$$

Therefore,

$$
\sum_{k=2}^{\infty} \lambda_{k}\left|f_{k}\right| \exp \left\{\eta \lambda_{k}\right\} \leq \frac{\lambda_{2}\left|f_{2}\right| \exp \left\{\eta \lambda_{2}\right\}+\frac{\lambda_{3}\left|\gamma_{0}\right|}{\lambda_{3}^{2}+\gamma_{2}} \exp \left\{\eta \lambda_{3}\right\}}{1-\frac{\lambda_{3}\left|\gamma_{1}\right| \exp \{h \eta\}}{\lambda_{2}\left(\lambda_{3}^{2}+\gamma_{2}\right)}-\frac{\lambda_{4}\left|\gamma_{0}\right| \exp \{2 h \eta\}}{\lambda_{2}\left(\lambda_{4}^{2}+\gamma_{2}\right)}}
$$

i. e. in view of (12) the inequality (13) holds.

3. Main result. The following theorem is true.

Theorem. Let $h>0, \gamma_{0} \neq 0, \gamma_{1} \neq 0, \gamma_{2}<0$ and for some $\eta \in \mathbb{R}$

$$
\frac{\left(\sqrt{\left|\gamma_{2}\right|}+2 h\right)\left|\gamma_{1}\right|}{4 h\left(\sqrt{\left|\gamma_{2}\right|}+h\right)^{2}} e^{h \eta}+\frac{\left(\sqrt{\left|\gamma_{2}\right|}+3 h\right)\left|\gamma_{0}\right|}{3 h\left(\sqrt{\left|\gamma_{2}\right|}+h\right)\left(3 h+2 \sqrt{\left|\gamma_{2}\right|}\right)} e^{2 h \eta}+
$$




$$
+\frac{\left|\gamma_{1}\right|\left(\sqrt{\left|\gamma_{2}\right|}+h\right) \exp \left\{\eta \sqrt{\left|\gamma_{2}\right|}\right\}}{h\left(h+\sqrt{\left|\gamma_{2}\right|}\right)} e^{h \eta}+\frac{\left(\sqrt{\left|\gamma_{2}\right|}+2 h\right)\left|\gamma_{0}\right| \exp \left\{\eta \sqrt{\left|\gamma_{2}\right|}\right\}}{4 h\left(\sqrt{\left|\gamma_{2}\right|}+h\right)} e^{2 h \eta} \leq 1
$$

Then the differential equation (4) has the entire solution (11) locally univalent in the halfplan

$$
\left\{s: \operatorname{Re} s<\min \left\{\eta, \frac{\eta\left(\sqrt{\left|\gamma_{2}\right|}+h\right)+\ln \sqrt{\left|\gamma_{2}\right|}}{h}\right\}\right\}
$$

and such that $\ln M(\sigma, F)=(1+o(1)) \frac{\sqrt{\left|\gamma_{0}\right|}}{h} e^{h \sigma}$ as $\sigma \rightarrow+\infty$.

Proof. The conditions (14) and (12) are equivalent. Therefore, by Lemma 2 the condition (13) holds and, thus, $F$ belongs to the class $\tau \geq \eta$. By Theorem B the function $F$ is locally univalent in half-plane $\left\{s: \operatorname{Re} s<\min \left\{\tau, \frac{\tau \lambda_{1}+\ln \lambda_{1} \mid}{\lambda_{2}-\lambda_{1}}\right\}\right\}$ and, thus, in half-plane (15).

Now we show that series (11) is entire. Indeed, since for every $\sigma \in \mathbb{R}$ there exists $k_{0}=$ $k_{0}(\sigma) \geq 3$ such that

we have, as above,

$$
\frac{\left|\gamma_{1}\right|}{\lambda_{k+1}^{2}+\gamma_{2}} e^{h \sigma}+\frac{\left|\gamma_{0}\right|}{l_{k+2}^{2}+\gamma_{2}} e^{2 h \sigma} \leq \frac{1}{2} \quad\left(k \geq k_{0}\right)
$$

$$
\begin{gathered}
\sum_{k=k_{0}}^{\infty}\left|f_{k}\right| \exp \left\{\sigma \lambda_{k}\right\} \leq \sum_{k=k_{0}-1}^{\infty} \frac{\left|\gamma_{1}\right|\left|f_{k}\right|}{\lambda_{k+1}^{2}+\gamma_{2}} \exp \left\{\sigma \lambda_{k}\right\} \exp \left\{\sigma\left(\lambda_{k+1}-\lambda_{k}\right)\right\}+ \\
+\sum_{k=k_{0}-2}^{\infty} \frac{\left|\gamma_{0}\right|\left|f_{k}\right|}{\lambda_{k+2}^{2}+\gamma_{2}} \exp \left\{\sigma \lambda_{k}\right\} \exp \left\{\sigma\left(\lambda_{k+2}-\lambda_{k}\right)\right\}= \\
=\frac{\left|\gamma_{1}\right|\left|f_{k_{0}-1}\right| \exp \left\{\sigma \lambda_{k_{0}}\right\}}{\lambda_{k_{0}}^{2}+\gamma_{2}}+\frac{\left|\gamma_{0}\right|\left|f_{k_{0}-2}\right| \exp \left\{\sigma \lambda_{k_{0}}\right\}}{\lambda_{k_{0}}^{2}+\gamma_{2}}+\frac{\left|\gamma_{0}\right|\left|f_{k_{0}-1}\right| \exp \left\{\sigma \lambda_{k_{0}-1}\right\}}{\lambda_{k_{0}-1}^{2}+\gamma_{2}}+ \\
+\sum_{k=k_{0}}^{\infty}\left(\frac{\left|\gamma_{1}\right|}{\lambda_{k+1}^{2}+\gamma_{2}} e^{h \sigma}+\frac{\left|\gamma_{0}\right|}{\lambda_{k+2}^{2}+\gamma_{2}} e^{2 h \sigma}\right)\left|f_{k}\right| \exp \left\{\sigma \lambda_{k}\right\} \leq \\
\leq \frac{\left|\gamma_{1}\right|\left|f_{k_{0}-1}\right| \exp \left\{\sigma \lambda_{k_{0}}\right\}}{\lambda_{k_{0}}^{2}+\gamma_{2}}+\frac{\left|\gamma_{0}\right|\left|f_{k_{0}-2}\right| \exp \left\{\sigma \lambda_{k_{0}}\right\}}{\lambda_{k_{0}}^{2}+\gamma_{2}}+\frac{\left|\gamma_{0}\right|\left|f_{k_{0}-1}\right| \exp \left\{\sigma \lambda_{k_{0}-1}\right\}}{\lambda_{k_{0}-1}^{2}+\gamma_{2}}+ \\
+\frac{1}{2} \sum_{k=k_{0}}^{\infty}\left|f_{k}\right| \exp \left\{\sigma \lambda_{k}\right\},
\end{gathered}
$$

whence it follows that $\sum_{k=k_{0}}^{\infty}\left|f_{k}\right| \exp \left\{\sigma \lambda_{k}\right\}<+\infty$, i. e. Dirichlet series (11) is absolutely convergent in $\mathbb{C}$.

For the study of the growth of (11) we use the Wiman-Valiron method. Let

$$
\mu(\sigma, F)=\max \left\{\left|f_{k}\right| \exp \left\{\sigma \lambda_{k}\right\}: k \geq 1\right\}
$$

be the maximal term of the Dirichlet series of form (5) and

$$
\nu(\sigma, F)=\max \left\{k:\left|f_{k}\right| \exp \left\{\sigma \lambda_{k}\right\}=\mu(\sigma, F)\right\}
$$

be its central index.

We suppose that the exponents of the Dirichlet series (5) satisfy the condition

$$
\int_{0}^{\infty} t^{-2} \ln n(t) d t<+\infty
$$


where $n(t)=\sum_{0<\lambda_{n} \leq t} 1$, and put

$$
\eta(x)=\int_{x}^{\infty} t^{-2} \ln n(t) d t, \quad l(x)=\frac{1}{\eta(x)} \ln ^{-2} \frac{1}{\eta(x)}, \quad k(x)=x \sqrt{1 / l(x)} .
$$

Then [6] for every $m \in \mathbb{N}$ and all $s, \operatorname{Re} s=\tau,|\tau-\sigma|<1 /\left(30 k\left(\lambda_{\nu}\right)\right)$,

$$
F^{(m)}(s)=\lambda_{\nu}^{m}(F(s)+o(M(\tau, F))), \quad \nu=\nu(\sigma, F),
$$

as $0 \leq \sigma \rightarrow+\infty$ outside of some set $E \subset[0+\infty)$ of finite measure and $E$ is contained in the union of intervals $\left[R_{\nu}+\tau_{\nu-1}, R_{\nu}+\tau_{\nu}\right)$ and $\tau_{\nu}-\tau_{\nu-1} \rightarrow 0$ as $\nu \rightarrow \infty$.

Let $\delta(\sigma)$ be an arbitrary positive function on $[0,+\infty)$, which tends to zero as $\sigma \rightarrow+\infty$, and $\Delta(\sigma)=\{s: \operatorname{Re} s=\sigma,|F(s)| \geq(1-\delta(\sigma)) M(\sigma, F)\}$. Then choosing $\tau=\sigma$ we obtain from $(16)$

$$
F^{(m)}(s)=\lambda_{\nu}^{m} F(s)(1+\varepsilon(\sigma)), \quad s \in \Delta(\sigma),
$$

where $\varepsilon(\sigma) \rightarrow 0$ as $\sigma \rightarrow+\infty, \sigma \notin E$.

Suppose that the Dirichlet series (11) satisfies (4). Substituting (17) in (4) we obtain $\lambda_{\nu}^{2}=\left|\gamma_{0}\right| e^{2 h \sigma}\left(1+\varepsilon_{1}(\sigma)\right)$, where $\varepsilon_{1}(\sigma) \rightarrow 0$ as $\sigma \rightarrow+\infty, \sigma \notin E$. Therefore,

$$
\lambda_{\nu(\sigma, F)}=(1+o(1)) \sqrt{\left|\gamma_{0}\right|} e^{h \sigma}, \quad \sigma \rightarrow+\infty, \sigma \notin E .
$$

If $\sigma \in E$, that is $\sigma_{\nu-1}=R_{\nu}+\tau_{\nu-1} \leq \sigma<\sigma_{\nu}=R_{\nu}+\tau_{\nu}$ for some $\nu$, then $\sigma_{\nu}-\sigma_{\nu-1} \rightarrow 0$ and, thus,

$$
e^{h \sigma_{\nu-1}}=(1+o(1)) e^{h \sigma}=(1+o(1)) e^{h \sigma_{\nu}}
$$

as $\nu \rightarrow \infty$. Therefore,

$$
\begin{gathered}
(1+o(1)) e^{h \sigma}=(1+o(1)) e^{h \sigma_{\nu-1}}=\lambda_{\nu\left(\sigma_{\nu-1}, F\right)} \leq \lambda_{\nu(\sigma, F)} \leq \lambda_{\nu\left(\sigma_{\nu}, F\right)}= \\
=(1+o(1)) e^{h \sigma_{\nu}}=(1+o(1)) e^{h \sigma}, \quad \sigma \rightarrow+\infty,
\end{gathered}
$$

i. e. (20) is true as $\sigma \rightarrow+\infty$. But [7, p. 182] ln $\mu(\sigma, F)=\ln \mu(0, F)+\int_{0}^{\sigma} \lambda_{\nu(t, F)} d t$. Therefore,

$$
\ln \mu(\sigma, F)=(1+o(1)) \frac{\sqrt{\left|\gamma_{0}\right|}}{h} e^{h \sigma}
$$

as $\sigma \rightarrow+\infty$ and, since $\ln \lambda_{n} \sim \ln n$ as $n \rightarrow \infty$, we have [8,9]

$$
\ln M(\sigma, F)=(1+o(1)) \ln \mu(\sigma, F), \quad \sigma \rightarrow+\infty .
$$

Suppose that $\gamma_{2} \leq-1$ and $\eta=0$. Then instead of (15) we have the half-plane

$$
\left\{s: \operatorname{Re} s<\min \left\{0,\left(\ln \sqrt{\left|\gamma_{2}\right|}\right) / h\right\}\right\}=\{s: \operatorname{Re} s<0\},
$$

and condition (14) has the form

$$
\frac{\left(\sqrt{\left|\gamma_{2}\right|}+2 h\right)\left|\gamma_{1}\right|}{4\left(\sqrt{\left|\gamma_{2}\right|}+h\right)^{2}}+\frac{\left(\sqrt{\left|\gamma_{2}\right|}+3 h\right)\left|\gamma_{0}\right|}{3\left(\sqrt{\left|\gamma_{2}\right|}+h\right)\left(3 h+2 \sqrt{\left|\gamma_{2}\right|}\right)}+\frac{\left|\gamma_{1}\right|\left(\sqrt{\left|\gamma_{2}\right|}+h\right)}{\left(h+\sqrt{\left|\gamma_{2}\right|}\right)}+\frac{\left(\sqrt{\left|\gamma_{2}\right|}+2 h\right)\left|\gamma_{0}\right|}{4\left(\sqrt{\left|\gamma_{2}\right|}+h\right)} \leq h .
$$

Since

$$
\frac{\sqrt{\left|\gamma_{2}\right|}+2 h}{4\left(\sqrt{\left|\gamma_{2}\right|}+h\right)^{2}} \leq \frac{1}{4}, \quad \frac{\sqrt{\left|\gamma_{2}\right|}+3 h}{3\left(\sqrt{\left|\gamma_{2}\right|}+h\right)\left(3 h+2 \sqrt{\left|\gamma_{2}\right|}\right)} \leq \frac{1}{3} \quad \text { and } \quad \frac{\sqrt{\left|\gamma_{2}\right|}+2 h}{4\left(\sqrt{\left|\gamma_{2}\right|}+h\right)} \leq \frac{1}{2},
$$

condition (19) holds if $\frac{5\left|\gamma_{1}\right|}{4}+\frac{5\left|\gamma_{0}\right|}{6} \leq h$, and the theorem implies the following corollary. 
Corollary. Let $h>0, \gamma_{0} \neq 0, \gamma_{1} \neq 0, \gamma_{2} \leq-1$ and $5\left|\gamma_{1}\right| / 4+5\left|\gamma_{0}\right| / 6 \leq h$. Then differential equation (4) has entire solution (11) locally univalent in the half-plane $\{s: \operatorname{Re} s<0\}$ and such that

$$
\ln M(\sigma, F)=(1+o(1)) \frac{\sqrt{\left|\gamma_{0}\right|}}{h} e^{h \sigma}
$$

as $\sigma \rightarrow+\infty$.

\section{REFERENCES}

1. G.M. Golusin, Geometrical theory of functions of complex variable, M.: Nauka, 1966, 628 p. (in Russian), Engl. transl.: AMS: Translations of Mathematical monograph, 26 (1969), 676 p.

2. W. Kaplan, Close-to-convex schlicht functions, Michigan Math. J., 1 (1952), №2, 169-185.

3. S.M. Shah, Univalence of a function $f$ and its successive derivatives when $f$ satisfies a differential equation, II. J. Math. Anal. and Appl., 142 (1989), 422-430.

4. P. Montel, Sur les fonctions localement univalentes ou multivalentes, Ann. Sci. Ecole Norm. Sup. (3), 54 (1937), 39-54.

5. M.S. Robertson, Schlicht Dirichlet series, Canad. J. Math., 10 (1958), 161-176.

6. M.N. Sheremeta, On the derivative of an entire Dirichlet series, Mat. sb., 137 (1988), №1, $128-139$ (in Russian); Engl. transl.: Math. USSR Sbornik, 65 (1990), №1, 133-145.

7. A.F. Leont'ev, Series of exponents, M.: Nauka., 1976, 536 p. (in Russian)

8. M.N. Sheremeta, Full equivalence of the logarithms of the maximum modulus and the maximal term of an entire Dirichlet series, Mat. Zametki, 47 (1990), №6, 119-123 (in Russian); Engl. transl.: Math. Notes, 47 (1990), №6, 119-123.

9. M.N. Sheremeta, On the correlations between the maximal term and the maximum modulus of an entire Dirichlet series, Mat. Zametki, 51 (1992), №5, 141-148 (in Russian); Engl. transl.: Math. Notes, 51 (1992), №5, 141-148.

Ivan Franko National University of Lviv

Lviv, Ukraine

m.m.sheremeta@gmail.com 